\title{
Transinguinal sonographic determination of the position of the femoral head after reposition and follow-up in a spica cast
}

\author{
Frederik J. A. Beek • Rutger-Jan Nievelstein • \\ Hans E. Pruijs • Pim A. de Jong • Ralph J. B. Sakkers
}

Received: 14 January 2010 /Revised: 14 April 2010 / Accepted: 27 April 2010 /Published online: 16 June 2010

(C) The Author(s) 2010. This article is published with open access at Springerlink.com

\begin{abstract}
Background Transinguinal sonography can be used to demonstrate the position of the femoral head after reduction of a dislocated hip.

Objective To determine whether transinguinal sonography can replace radiography and $\mathrm{CT}$ in the follow-up after reduction of a hip dislocation?

Materials and methods Thirty-three children with 39 dislocated hips were followed up with sonography after reduction and immobilization in a spica cast. In cases of an abnormal position a CT scan was advised. A pelvic radiograph at the end of treatment served as an indicator that no dislocations were missed during the previous sonographic examinations.

Results The repositioned hips were examined on 138 occasions. Twenty-four examinations were abnormal and CT scanning was performed on 11 occasions. In four children additional CT was done because a recurrent dislocation was suspected or because sonography was difficult to perform. No dislocations were demonstrated. In five children a recurrent dislocation was suspected, on one or more occasions. In all but one child a CT scan was performed that confirmed the dislocation.
\end{abstract}

F. J. A. Beek $(\bowtie) \cdot$ R.-J. Nievelstein · P. A. de Jong

Department of Radiology, Wilhelmina Children's Hospital,

University Medical Centre Utrecht,

E.01.231, Heidelberglaan 100,

3584 Utrecht, The Netherlands

e-mail: e.beek@umcutrecht.nl

H. E. Pruijs · R. J. B. Sakkers

Department of Orthopedic Surgery,

Wilhelmina Children's Hospital,

University Medical Centre Utrecht,

Utrecht, The Netherlands
Conclusion Transinguinal sonography is well-suited to demonstrate a normal position of the femoral head in a spica cast. Transinguinal sonography decreases the number of radiographs and $\mathrm{CT}$ scans and reduces the exposure to ionizing radiation.

Keywords Developmental dysplasia of the hip . Hip dislocation · Spica cast treatment $\cdot$ US · Child

\section{Introduction}

Developmental dysplasia of the hip (DDH) is a common disorder of newborns and infants. It can have serious consequences. Approximately $30 \%$ of all individuals who undergo hip replacement before the age of 60 years have been diagnosed with DDH [1]. The goals of early recognition and treatment of DDH are to stimulate normal development of the dysplastic hip as much as possible and to prevent early hip degeneration. Therefore, in patients with a frank dislocation, the first goal is to reduce the femoral head. For that purpose abduction splinting is usually first applied. However, if the dislocation persists a closed or open reduction in the operating room is performed and the reduced hip is subsequently immobilised by using a spica cast. Both in the operating room and during follow-up the position of the hip needs to be frequently monitored with radiological techniques. Conventional radiography, CT, MRI and US are all possible for this purpose. Radiographs have been used to monitor patients with reduced hips in casts. The main disadvantage is the difficult interpretation because of the superposition of the spica cast (most often plaster of Paris) [2]. If the radiograph is unclear, additional imaging can be done. CT scans can nicely demonstrate the position of the femoral 
heads, but the radiation dose makes CT a less attractive routine test [3]. MRI can also depict the position of the hip. This can be done without sedation or anaesthesia, because motion artefacts are limited in a spica cast $[4,5]$. The experience with MRI, however, is limited and the costs are higher than for sonography. In addition, $\mathrm{CT}$ and MRI are not possible in the operating room.

Sonography, however, has great advantages. Firstly, it is an inexpensive technique that does not use X-rays. Secondly, it can be used to image the position of the femoral head directly after reduction while the child is still under anaesthesia in the operating room. If the reduction is unsuccessful a second reduction can be done. After application of the spica cast it can establish if the reduction is maintained. Thirdly, it is possible to monitor the position of the femoral head frequently in the spica cast at low cost and no radiation dose. Lastly, although sonography is observer-dependent, it is fairly easy to evaluate the position of the femoral head. Several reports regarding sonographic evaluation were published, mostly in the orthopaedic literature [6-8]. This application of sonography was termed "transinguinal sonography" [2]. Unfortunately, this technique is not very familiar to paediatric radiologists. We use sonography routinely during reduction under general anaesthesia in children in spica casts during follow-up and in children in an abduction splint like the Pavlik harness. In the present report we aim to familiarize paediatric radiologists with the transinguinal sonography technique to reduce radiation and costs for these children. We illustrate and discuss the technique and report our experience from the outpatient follow-up for the children in casts that we followed during the last 5 years.

\section{Materials and methods}

\section{Patients}

The records of all children with DDH who underwent open or closed reduction for persistent dislocation of the hip were reviewed. These children undergo sonography in the operating theatre and during follow-up routinely at our hospital. CT scans are obtained when clinically indicated. Radiographs are obtained after removal of the spica cast at the end of treatment. We recorded the number of follow-up sonographic examinations, the position of the femoral heads and the number of additional CT scans. In children with unilateral dislocation we only report on the results of the dysplastic dislocated hips. Syndromic patients suspected of teratologic dislocations were excluded.

This retrospective investigation was approved by the ethical review board and informed consent was waived.
Sonographic examination

For the sonographic follow-up the applied plaster of Paris cast must leave a slightly larger area of the perineum uncovered to create adequate space for the sonography transducer (Fig. 1). The sonographic examination is performed using a 5- to 8$\mathrm{MHz}$ or 4- to 9-MHz convex transducer, or a 5- to $12-\mathrm{MHz}$ linear-array transducer, depending on the size of the opening in the spica cast and/or the size of the child. A HDI 5000 or IU 22 US machine (Philips Healhcare, Best, The Netherlands) was used in this study.

During sonographic examination, the transducer is orientated in the transverse plane and first the symphysis pubis is located. Next, the transducer is moved laterally over the superior pubic ramus until the hip joint is visible. If a normal relation between femoral head and acetabulum is present the femoral head is easily seen resting against the cartilaginous surface of the acetabulum. The superior pubic ramus has to be in line with the femoral neck (Fig. 2).

In the first weeks after reduction some echogenic material can be visible between acetabulum and femoral head (Fig. 3) and is considered normal. This echogenic material disappears over time.

In this study, the position of the femoral head was labelled as abnormal if the femoral neck was not in line with the superior pubic ramus, which was the only US criterion that was used in this study. In cases that presented for the first time with such a US finding the referring orthopaedic surgeon was consulted and further steps in imaging or treatment were discussed. In most cases a CT scan of the acetabular region was performed to determine the exact position. If the position was normal and stable at follow-up no additional CT scans were performed.

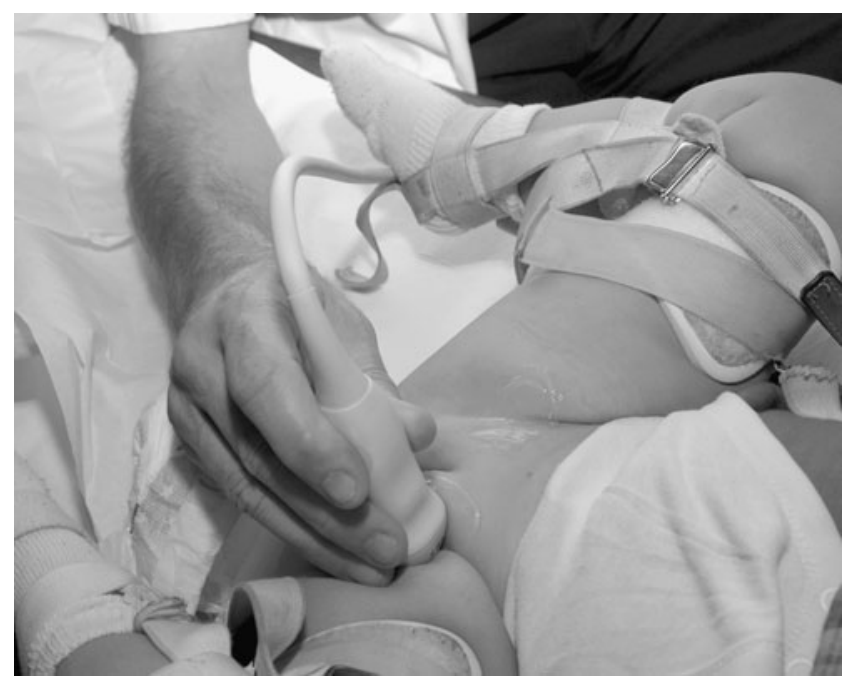

Fig. 1 Clinical photograph shows transinguinal sonography of the left hip. A convex transducer with a small footprint is orientated in the transverse plane 


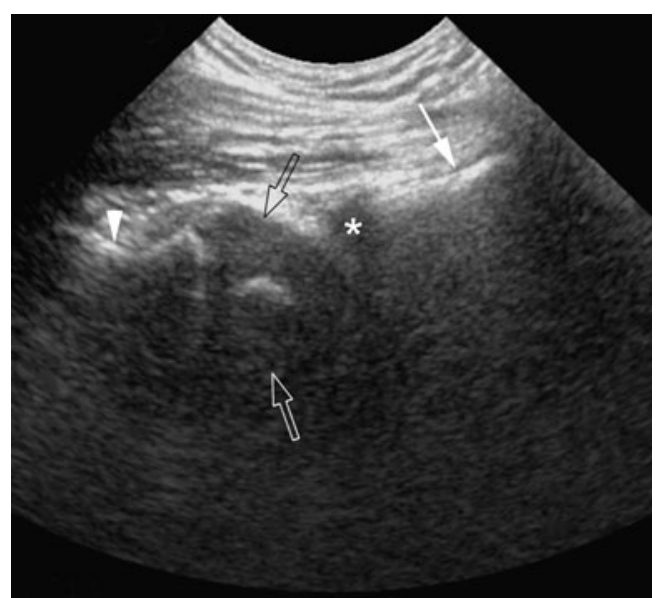

Fig. 2 Sonogram of a normal right hip of a 9-month-old girl in a spica cast. The superior pubic ramus (white arrow), the cartilaginous rim of the acetabulum (asterisk), the ossified femoral metaphysis (arrowhead) and the femoral head with a small ossified nucleus are visible (open arrows)

CT scanning was done on a Philips Brilliance 16P (Philips, Best, The Netherlands) using the following protocol: $90 \mathrm{kVp}, 30 \mathrm{mAs}, 16$ 1.5-mm detectors. The scan starts approximately $2 \mathrm{~cm}$ above the femoral head and ends below the femoral neck. This CT protocol results in an effective dose of $0.4 \mathrm{mSv}$. A pelvic radiograph has an effective dose of approximately $0.02 \mathrm{mSv}$.

After removal of the cast a pelvic radiograph was taken to assess the status of the hip joint. This radiograph served as proof that no dislocations were missed during the previous sonographic examinations.

\section{Results}

We included 33 children (29 girls, 4 boys) with 39 dislocated hips that were reduced in the operating theatre and immobilised in spica casts. The average age at reduction was 14 months, range $7-31$ months. Six children had bilateral hip dislocation. Of the 27 children with unilateral dislocations, 20 had left-side and 7 had rightside dislocation. Ten reductions were open and 29 closed. The 39 dislocated hips in 33 children were examined during 138 sonographic studies (165 hip evaluations). The average number of sonographic examinations was 4.1 , with multiple exams performed twice $(n=2)$, three- $(n=10)$, four- $(n=12)$, five- $(n=4)$, seven- $(n=4)$ and eight $(n=1)$ times.

In 24 children ( 3 with bilateral dislocation, 27 hips) the repositioned hips were in an adequate position during the follow-up examinations (89 hip examinations). No additional imaging was done in these cases.

In the other 9 children ( 3 with bilateral dislocation, 12 hips, 65 examinations) some follow-up sonographic evaluations were inconclusive or abnormal as described in Table 1. In this group $11 \mathrm{CT}$ scans were made for further evaluation.

In 1 child the hole in the spica cast was too small to allow a proper examination and therefore this case was followed up with plain radiographs. In two cases CT showed that the femoral head was located more posterior than usual but still in the acetabulum. They were followed up with sonography. In one case with an inconclusive sonographic examination on day 1 , CT demonstrated a normal position.

In five children, seven repeat dislocations were found and the children were re-operated. Five dislocations were confirmed with CT, two were re-operated based on the sonographic findings.

The course of patient 5 with a unilateral dislocation was unusual. The dislocation persisted after reduction and this was demonstrated on four successive sonographic examinations. The orthopaedic surgeon tried to reduce the hip by filling up the spica cast behind the buttock. The fourth sonographic examination showed an anterior dislocation of the femoral head that was confirmed by CT (Fig. 4). The
Fig. 3 a Sonogram of the left hip of a 10-month-old girl, 2 days after closed reduction. The superior pubic ramus (white $a r$ row), the ossified femoral metaphysis (arrowhead) and the femoral head with a small ossified nucleus are visible (open arrow). Some echogenic material is seen between the hypoechoic cartilaginous acetabular rim and the femoral head (asterisk). The femoral neck and the superior pubic ramus are in line. b Same hip, 6 weeks later. The echogenic material (asterisk) has almost disappeared
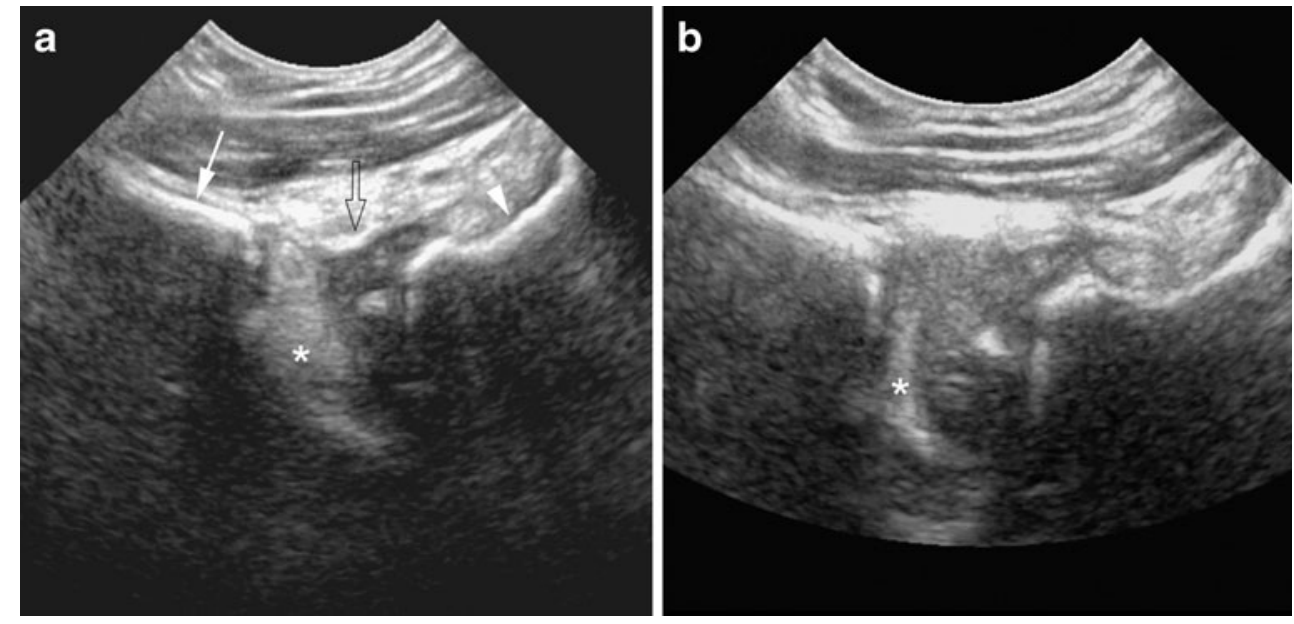
Table 1 List of complicated cases that required additional CT examination and/or re-operation

\begin{tabular}{|c|c|c|c|c|c|c|c|}
\hline Patient & Age (months) & $\begin{array}{l}\text { Uni-/bi-lateral } \\
\text { dislocation }\end{array}$ & $\begin{array}{l}\text { Number of } \\
\text { US per } \\
\text { patient }\end{array}$ & $\begin{array}{l}\text { Number of } \\
\text { imaged } \\
\text { diseased hips }\end{array}$ & $\begin{array}{l}\text { Number of } \\
\text { abnormal } \\
\text { US }\end{array}$ & $\begin{array}{l}\text { Number of } \\
\text { CT scans per } \\
\text { patient }\end{array}$ & Comments \\
\hline 1 & 21 & Unilateral & 2 & 2 & 2 & 1 & $\begin{array}{l}\text { Too small hole in plaster, further } \\
\text { follow-up with plain radiographs }\end{array}$ \\
\hline 2 & 30 & Unilateral & 7 & 7 & 2 & 1 & $\begin{array}{l}\text { Recurrent dislocation at day } 30 . \\
\text { Re-operation }\end{array}$ \\
\hline 3 & 11 & Unilateral & 8 & 8 & 3 & 1 & $\begin{array}{l}\text { Twice recurrent dislocation at day } \\
20 \text { and } 35 \text {. Twice re-operation }\end{array}$ \\
\hline 4 & 11 & Unilateral & 4 & 4 & 1 & 1 & $\begin{array}{l}1 \text { day postoperative US indeterminate. } \\
\text { CT showed correct position }\end{array}$ \\
\hline 5 & 9 & Unilateral & 7 & 7 & 4 & 1 & $\begin{array}{l}\text { CT after } 4 \text { abnormal US examinations } \\
\text { Re-operation at day } 28\end{array}$ \\
\hline 6 & 31 & Unilateral & 5 & 5 & 5 & 2 & $\begin{array}{l}\text { Femoral head on posterior acetabular } \\
\text { rim at every US and CT examination } \\
\text { Expectant management }\end{array}$ \\
\hline 7 & 31 & Bilateral & 4 & 8 & 4 & 2 & $\begin{array}{l}\text { Right femoral head on posterior } \\
\text { acetabular rim at every US and CT } \\
\text { examination. Expectant management }\end{array}$ \\
\hline 8 & 11 & Bilateral & 5 & 10 & 1 & 0 & $\begin{array}{l}\text { Recurrent dislocation of right hip at } \\
\text { day } 1 . \text { Re-operation with uneventful } \\
\text { follow-up }\end{array}$ \\
\hline 9 & 12 & Bilateral & 7 & 14 & 2 & 2 & $\begin{array}{l}\text { Twice recurrent dislocation of left hip } \\
\text { at day } 28 \text { and } 62 \text {. Twice re-operation }\end{array}$ \\
\hline
\end{tabular}

child was than re-operated with a good result and immobilized in an abduction splint.

At the end of treatment, in all 33 children the hips were shown to be adequately reduced on the radiographs immediately after removal of the spica cast.

\section{Discussion}

After reposition of a dislocated femoral head in children with DDH the position of the femoral head needs to be followed with imaging. Conventional radiography, CT, MRI, and sonography are possible tests, but in our opinion the preferred method is sonography. In the 33 children that we evaluated during the last 5 years, a good position of the femoral head at the end of cast therapy was achieved in all, when monitoring mainly consisted of sonography and an end-of-treatment radiograph after the cast was removed. For the 33 children (39 hips) we only needed $11 \mathrm{CT}$ scans in 8 children to confirm sonographic findings.

The application of sonography in this patient group was described in 1988 by Keller and Weiss [6] as a case report. They described sonography during reduction of a dislocated hip in the operating room. In 1991 Suzuki et al. [7] described this method using a linear-array probe with a large field of view. With that probe both hips were imaged
Fig. 4 a Sonogram of the right hip of an 11-month-old girl. The femoral head (open arrow) is too low and too anterior in relation to the ischial bone (arrow). This is confirmed on axial CT (b)
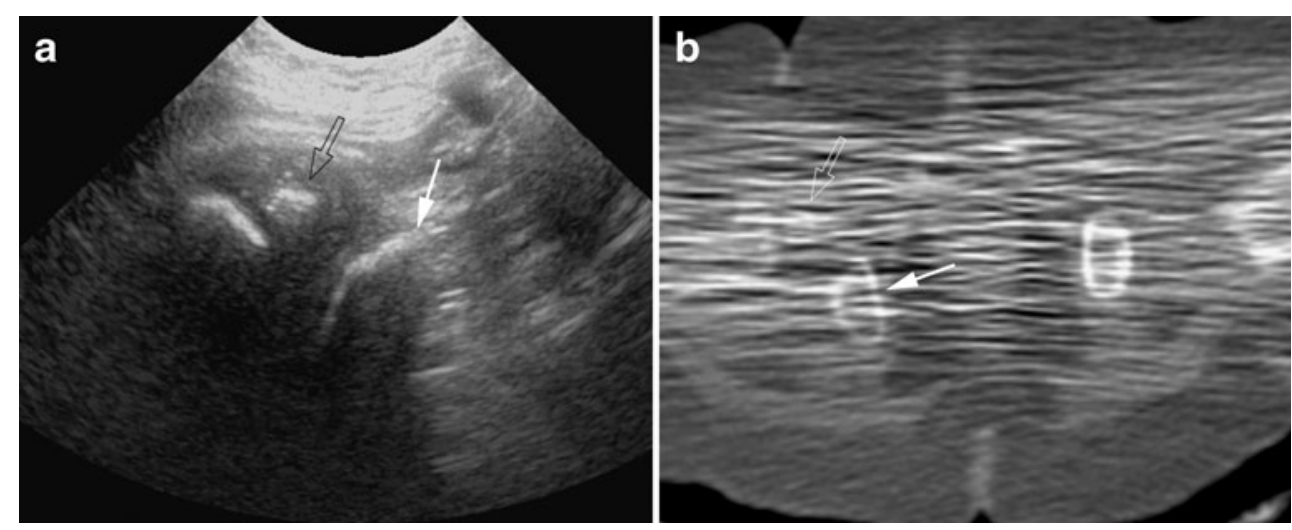
simultaneously. In 2003 van Douveren et al. [8] described sonography after closed or open reduction in the operating room. Those authors compared sonographic findings to CT, which was performed a few minutes later. The correlation between sonographic findings and CT findings was excellent. Recently, Eberhardt et al. [2] published a comparison between sonography and radiographs. They concluded that the sonographic method was far superior to radiography, because the superimposition of the cast hampers the interpretation of radiographs. Most of these studies were published in the orthopaedic literature and the method seems to be not well known by paediatric radiologists.

Our study shows that sonography can display the correct position of the femoral head, with the femoral neck in line with the superior pubic ramus, in the follow-up after reduction of a dislocation with application of a spica cast. If sonography shows an adequate position of the femoral head no other imaging is necessary. All children with normal sonography had a good post-treatment result as assessed by radiography. If the femoral head is not detected in an adequate position it can be completely dislocated, but it can also rest against the posterior acetabular roof (Fig. 5)

In eight children an abnormal position was shown. In one child the position was normal on CT and this is a falsepositive result. In two children the femoral head was located posteriorly in the acetabulum (Table 1). In five children the abnormal position of the femoral head represented a redislocation and a redislocation occurred twice in two of these five cases. In five of these events a CT scan was done and on two occasions the orthopaedic surgeon re-operated based on the sonographic results. After the re-operation the sequence of sonographic follow-up started again, leading to quite a number of sonographic examinations in some children (Table 1). On average four sonographic examinations per child were done. In one child without redislocation seven examinations were performed. This girl was referred from an outside hospital after failed treatment in a spica cast and she was followed-up closely with five sonographic exams in the first 2 weeks and two subsequent examinations. The reduction was successful.

With sonography it is apparently difficult to determine the exact position of the femoral head in relation to the posterior rim of the acetabulum. This could be due to the deeper position of the posterior rim relative to the anterior rim. Also, there is always some echogenic material between the acetabulum and the reduced femoral head and this could hamper imaging of the posterior acetabular rim. Because of this difficulty we recommend additional CT imaging if the femoral neck is not in line with the superior pubic ramus.

The described sonographic technique is easily learned and can quickly be mastered by radiologists, residents and sonographers. If the hip is well-positioned this will be evident within a minute. If the hip is malpositioned one should try to determine the position of the femoral head. If the femoral head is anteriorly displaced, which it seldom is, it can be easily visualized. In the more common posterior position, it is often very difficult or impossible to actually depict the femoral head itself. However, starting with imaging at the femoral shaft, it can be shown that the femoral neck projects posterior, although the femoral head itself is hidden in the gluteal muscles.

Our results are in line with the study of Eberhardt et al. [2]. In that study sonography and radiographs were compared in 25 children on 68 occasions. It showed that retrospectively $47 \%$ of the radiographs were insufficient for exactly determining the femoral head position and that all US examinations correctly demonstrated the position of the reduced hip. In the study of Eberhardt the average age of the children was 6.5 weeks, considerably younger than in our study where the average age was 14 months. Our study shows that older age is not a limiting factor for transinguinal sonography.

One could argue that we used radiography as the "reference standard" after removal of the spica cast. When we implemented transinguinal sonography in our practice we compared the sonographic results with CT scans [8]. The results showed that sonography was sufficient to
Fig. 5 a Sonogram in a 2.5-year-old girl with bilateral dislocation. The right femoral head is not well depicted at the level of the superior pubic ramus (arrow). Only some echogenic material is visible. The femoral neck is seen deeper in the soft tissues (arrowhead) and not in line with the superior pubic ramus. b CT scan shows that the femoral head (open arrow) is dorsally positioned in the acetabulum
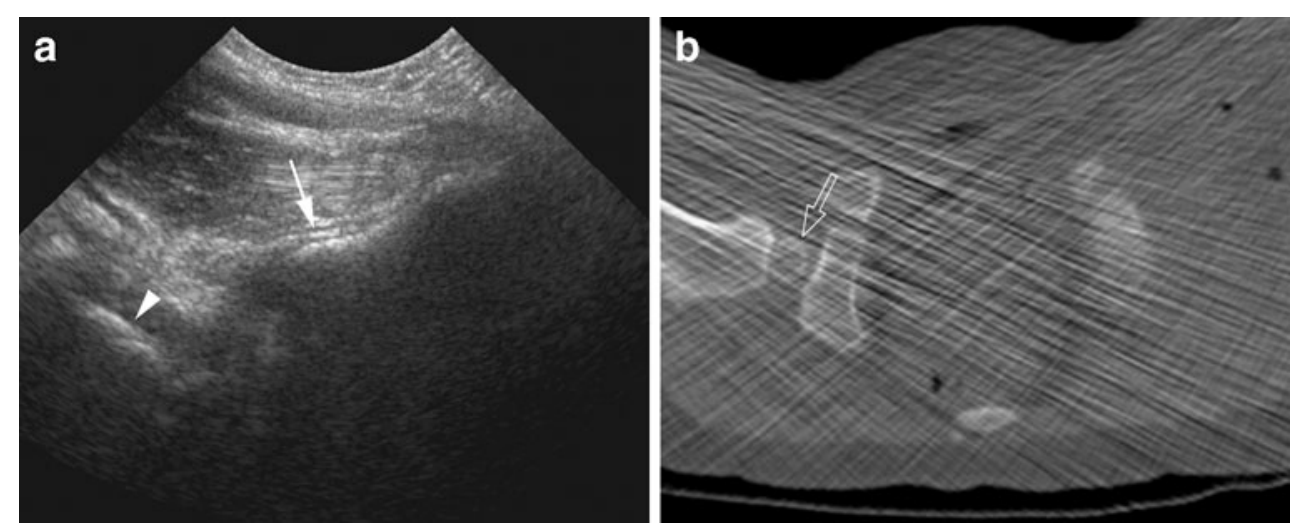
demonstrate a normal position of the femoral head. Thereafter we applied CT imaging only in children in whom the sonographic findings were unclear or abnormal. We consider a radiograph with an adequate position of the hips sufficient to demonstrate a satisfactory reduction.

Several problems can be encountered during sonography in a spica cast. First of all the opening in the cast over the lower body orifices needs to be large enough laterally to provide space for the US probe. Communication with the orthopaedic surgeons applying the cast is mandatory to assure that the opening is sufficient. Secondly, the examination can be difficult during or shortly after open operative reduction due to interference by collections of air in the soft tissues. Furthermore, as discussed above it can be difficult or impossible to detect the actual position of a femoral head that is located more posteriorly than normal. It can be either posteriorly positioned in the acetabulum or frankly dislocated. When in doubt, CT may be indicated to determine the exact position of these hips. Finally, after reduction an echogenic rim of tissue is almost invariably seen between the acetabulum and the repositioned femoral head. On CT this is shown to consist of interposed fatty tissue, also named pulvinar [8]. The normal hip does not show this interposed echogenic tissue. This tissue disappears in the course of a few weeks.

\section{Conclusion}

Transinguinal sonography is an easy technique and wellsuited to demonstrate a normal position of the femoral head in a spica cast. At our hospital, radiography, CT and MRI are not routinely used in this patient group, but only when indicated after sonography. Transinguinal sonography decreases the exposure to ionizing radiation in this category of patients likely to be subjected to more radiation-mediated imaging in their lifetime.

Open Access This article is distributed under the terms of the Creative Commons Attribution Noncommercial License which permits any noncommercial use, distribution, and reproduction in any medium, provided the original author(s) and source are credited.

\section{References}

1. Furnes O, Lie SA, Espehaug B et al (2000) Hip disease and the prognosis of total hip replacements. A review of 53, 698 primary hip replacements reported to the Norwegian Arthroplasty Register 1987-99. J Bone Joint Surg Br 83:579-586

2. Eberhardt O, Zieger M, Langendoerfer M et al (2009) Determination of hip reduction in spica cast treatment for DDH: a comparison of radiography and ultrasound. J Child Orthop 3:313-318

3. Mandel DM, Loder RT, Hensinger RN (1998) The predictive value of computed tomography in the treatment of developmental dysplasia of the hip. J Pediatr Orthop 18:794-798

4. Laor T, Roy DR, Mehlman CT (2000) Limited magnetic resonance imaging examination after surgical reduction of developmental dysplasia of the hip. J Pediatr Orthop 20:572-574

5. Ranawat V, Rosendahl K, Jones D (2009) MRI after operative reduction with femoral osteotomy in developmental dysplasia of the hip. Pediatr Radiol 39:161-163

6. Keller MS, Weiss AA (1988) Sonographic guidance for infant hip reduction under anesthesia. Pediatr Radiol 18:174-175

7. Suzuki S, Kasahara Y, Futami T et al (1991) Ultrasonography in congenital dislocation of the hip. Simultaneous imaging of both hips from in front. J Bone Joint Surg Br 73:879-883

8. Van Douveren FQ, Pruijs HE, Sakkers RJ et al (2003) Ultrasound in the management of the position of the femoral head during treatment in a spica cast after reduction of hip dislocation in developmental dysplasia of the hip. J Bone Joint Surg Br 85:117-20 